\title{
Acute Vestibulopathy
}

\author{
The Neurohospitalist \\ I(I) $32-40$ \\ (C) The Author(s) 2011 \\ Reprints and permission: \\ sagepub.com/journalsPermissions.nav \\ DOI: I0.1 I77/I94I875210386235 \\ http://nhos.sagepub.com
}

()SAGE

Yoon-Hee Cha, MD'

\begin{abstract}
The presentation of acute vertigo may represent both a common benign disorder or a life threatening but rare one. Familiarity with the common peripheral vestibular disorders will allow the clinician to rapidly "rule-in" a benign disorder and recognize when further testing is required. Key features of vertigo required to make an accurate diagnosis are duration, chronicity, associated symptoms, and triggers. Bedside tests that are critical to the diagnosis of acute vertigo include the Dix-Hallpike maneuver and canalith repositioning manuever, occlusive ophthalmoscopy, and the head impulse test. The goal of this review is to provide the clinician with the clinical and pathophysiologic background of the most common disorders that present with vertigo to develop a logical differential diagnosis and management plan.
\end{abstract}

\section{Keywords}

vertigo, Dix-Hallpike, BPPV, Meniere disease, vestibular neuritis

\section{Introduction}

Dizziness ranks as one of the most common reasons why patients present for medical care. Vertigo, a true perception of environmental motion, is one distinct subtype of dizziness. This review will cover the most common peripheral and central vestibular disorders that present with rotational vertigo. A good history to exclude metabolic causes such as medications, cardiovascular disease, orthostasis, and psychogenic causes is presumed. Most vestibular disorders can be diagnosed with a careful history and bedside tests that do not require any specialized equipment. Basic clinical features such as duration, chronicity, associated auditory or neurologic features, and triggers will lead to a diagnosis in most cases (Table 1). When a patient presents with unusual symptoms or signs that do not fit into one of the benign disorders, further testing with imaging or specialized vestibular function testing may be required.

\section{Benign Paroxysmal Positional Vertigo}

Benign paroxysmal positional vertigo (BPPV) occurs with a lifetime prevalence of $2.4 \%{ }^{1}$ Although common, the symptoms can be so frightening that the patient's first encounter for treatment may be in the emergency room. Patient characteristics associated with BPPV are older age, history of head trauma, inner ear surgery, other inner ear disease, prolonged recumbency, and in younger affected patients, a history of migraine headaches. ${ }^{1-6}$

Benign paroxysmal positional vertigo is caused by otolith particles that become loosened from the sensory receptor of the utricle called the macula. ${ }^{7}$ Since these particles are heavy, they fall into the most dependent portion of the semicircular canal system, which is the posterior semicircular canal (PSC). Once they fall in, they get trapped until a liberatory manuever can be done. Freely moving otolith particles trapped in the semicircular canals is called canalithiasis and is the basis for BPPV. ${ }^{7,8}$

The PSC is affected in about $85 \%$ to $90 \%$ of cases, the horizontal semicircular canal (HSC) in $10 \%$ to $13 \%$, and the anterior canal in $1 \%$ to $2 \%$ of cases. ${ }^{9-11}$ The symptoms of BPPV occur because the otoliths, which are heavier than the surrounding endolymphatic fluid, move when the head is moved in relation to gravity or is moved in the plane of the affected canal. The movement of the endolymph causes the cupula, the angular acceleration-detecting receptor, to be deflected, which stimulates the hair cells and generates a neural signal that creates the perception that one side of the head is moving whereas the other (normal) side is not. The patient thus experiences rotational vertigo. The typical situations in which vertical head movements are made are when patients get up from bed, lie down on a bed, or extend their head (top shelf vertigo). The particles will also move if the head is rolled in the plane of the affected canal. Since the PSC sits at a $45^{\circ}$ angle relative

' UCLA Department of Neurology, Los Angeles, CA, USA

Corresponding Author:

Yoon-Hee Cha, MD, UCLA Department of Neurology, 710 Westwood Plaza Box 951769, Los Angeles, CA 90095, USA

Email: yhcha@mednet.ucla.edu 


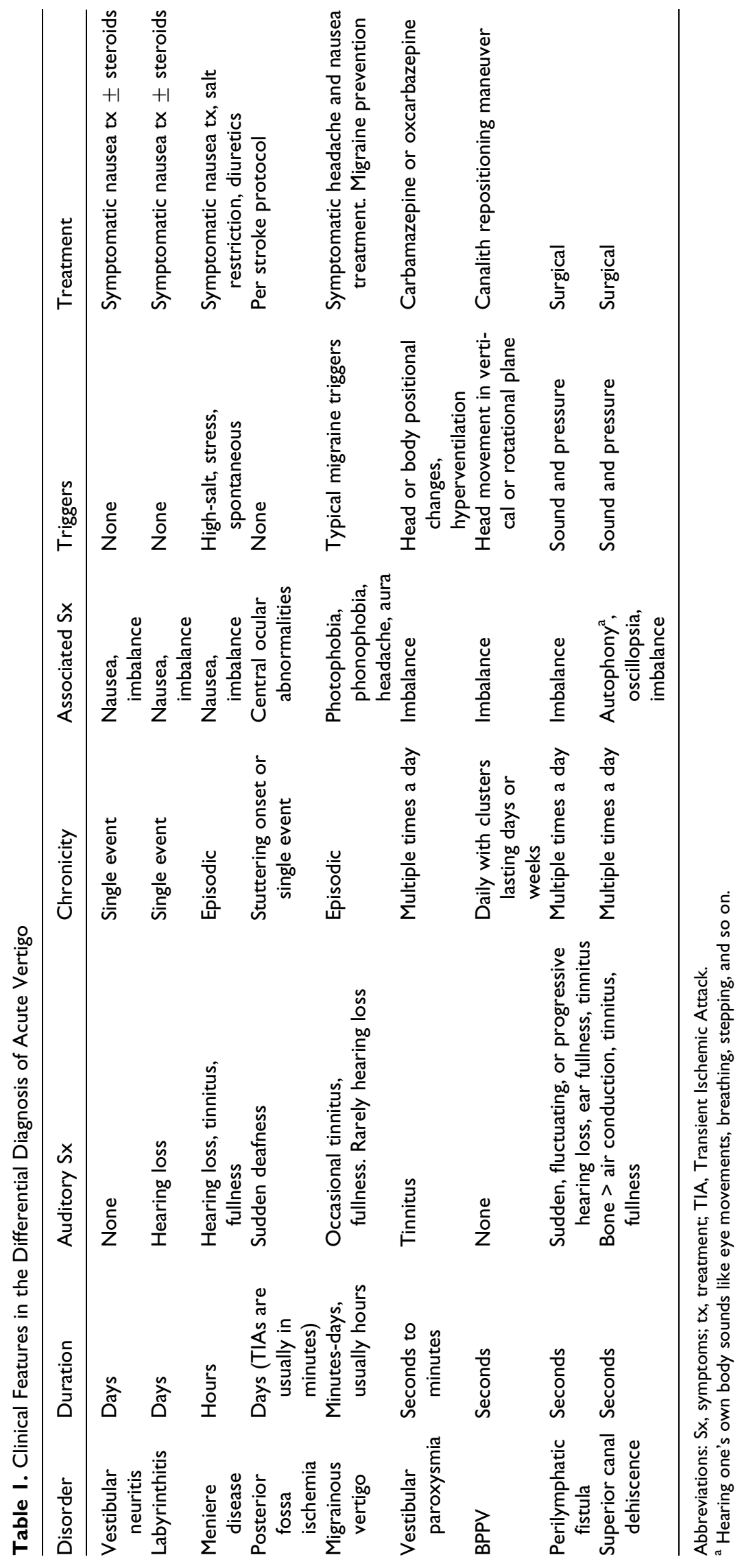


to the midline, rolling toward the affected ear will also trigger spells.

The classic features of BPPV are very brief episodes (seconds) of vertigo that are solely triggered by head movement. This head movement is in the vertical or rotational plane. If patients complain of vertigo only while standing, turning their bodies without turning their heads, or turning their heads in the horizontal plane only, another diagnosis should be considered. It should also be noted that patients with any kind of central or peripheral cause of vertigo may feel worse when they move their heads because any kind of baseline asymmetry in vestibular function will be ampliflied by head movement. Patients with severe spells will sometimes feel off-balanced for a brief time after a severe vertigo episode. They may also have nausea. However, patients with BPPV should have no gait problems in between spells as very little vertical head movements are done when standing and walking. They should also be free from any other cranial symptoms.

Benign paroxysmal positional vertigo of the PSC is diagnosed with the Dix-Hallpike maneuver, a maneuver that places the patient's head in the plane of the affected PSC to maximally mobilize the otoliths trapped in that canal (Figure 1, steps 1 and 2). The side to which the patient turns that triggers the spell is the affected side. In this maneuver, the patient is placed in the sitting position with his or her side to the examiner. The head is turned $45^{\circ}$ to the side. The side to which the patient turns is the side being tested. The patient's head is lowered so that it hangs below the horizontal plane. If there are freely moving otoliths in the PSC, this maneuver will trigger nystagmus and vertigo.

The PSC is connected through brain stem nuclei that innervate the ipsilateral superior oblique and the contralateral inferior rectus muscles. ${ }^{12}$ The eye movement that is stimulated is a torsional vertical movement where the ipsilateral eye is depressed and intorted and the contralateral eye is depressed and extorted. The corrective movement, or saccade is thus an oppositely directed torsional vertical movement where the ipsilateral eye is raised and extorted and the contralateral eye is raised and intorted. Thus, when the right PSC is affected and a Dix-Hallpike is done to the right-hand side, the nystagmus elicited is a torsional vertical nystagmus that beats counterclockwise toward the top of the head. The nystagmus will appear as a burst after an initial variable delay, which is rarely as long as 30 seconds. The nystagmus will stop once the particles stop moving and the cupular deflection recovers. For the PSC, this is always less than 1 minute and generally closer to 15 to 20 seconds. ${ }^{13,14}$ Repeated testing will cause the response to fatigue.

Benign paroxysmal positional vertigo is treated with a canalith repositioning maneuver (CRM) also known as the modified Epley maneuver. The goal is to return the particles to the utricle where they redissolve back into the endolymphatic fluid. The initial steps of the CRM are the same as the DixHallpike manuever. At the end of the Dix-Hallpike maneuver, when the patient's vertigo and nystagmus have stopped, any loose otoliths will be settled at the apex of the PSC. Then, with the patient's head still hanging, a smooth roll to the opposite side is made followed by an additional body turn such that the patient ends up lying on his or her side (Figure 1, steps 3 and 4). The patient's view is now toward the floor. The patient may experience another short bout of vertigo; if the eyes are viewed at this time, they should still have the same direction of nystagmus as was originally seen during the Dix-Hallpike maneuver. From the standpoint of the canal, all that is done in this step is to rotate the PSC on its apex such that the dependent limb (through which the otoliths have just traveled) is now the upper limb. The patient is then brought back to a sitting position. With this step, the particles will fall down from the upper limb back into the utricle. The patient should be supported for about a minute after the procedure because if any otoliths suddenly fall onto the macula of the utricle, the patient will feel like they are being pushed and can fall off the examination table. This is extremely frightening but is actually reassuring that the otoliths have successfully been moved back into the utricle. A confirmatory Dix-Hallpike maneuver should be performed to ensure that the otoliths have all been rolled out. If positive, another CRM is performed until a negative confirmatory Dix-Hallpike maneuver is achieved. Since this is a treatment, and not an exercise, the patient does not need to continue performing the maneuver unless they have a symptom recurrence. Activity restrictions such as sleeping with a neck collar or sleeping upright are no longer considered necessary. ${ }^{15,16}$ However, patients should be instructed to avoid prolonged head extension as the particles can fall back out again. The rate of recurrence is estimated to be about $50 \%$ in 10 years, with most recurrences $(80 \%)$ occuring within the first year. ${ }^{17}$ Benign paroxysmal positional vertigo associated with trauma or baseline inner ear dysfunction has a higher recurrence rate. ${ }^{18,19}$ Left untreated, PSC BPPV resolves with a median duration of 39 days and HSC BPPV in 1 day. ${ }^{20}$

Benign paroxysmal positional vertigo of the horizontal canal is less common, but clinicians should keep this entity in mind if the patient develops much more severe vertigo after having the standard CRM done, or has head movement triggered vertigo that lasts up to a minute. ${ }^{21}$ It is diagnosed by eliciting geotropic beating nytagmus (fast-phase beating toward the undermost ear) when the patient is placed in the supine position with the head turned to either side. The time constant of recovery of the cupula of the HSC is much longer than that of the PSC, so when particles get trapped in this canal (which can happen during a CRM for the PSC since the 2 canals are connected before joining the vestibule), the vertigo spells are much longer. ${ }^{22}$ It can often be treated conservatively by having the patient sleep on the unaffected ear for a few nights. Specific liberatory maneuvers like the Lempert-BBQ roll maneuver or the Gufoni maneuver may also be performed. ${ }^{23-25}$

On occasion, a patient may not be able to perform the headhanging CRM because of poor neck extension or immobility. An alternative procedure is the Semont maneuver, in which the patient turns $45^{\circ}$ toward the unaffected ear and lies down 


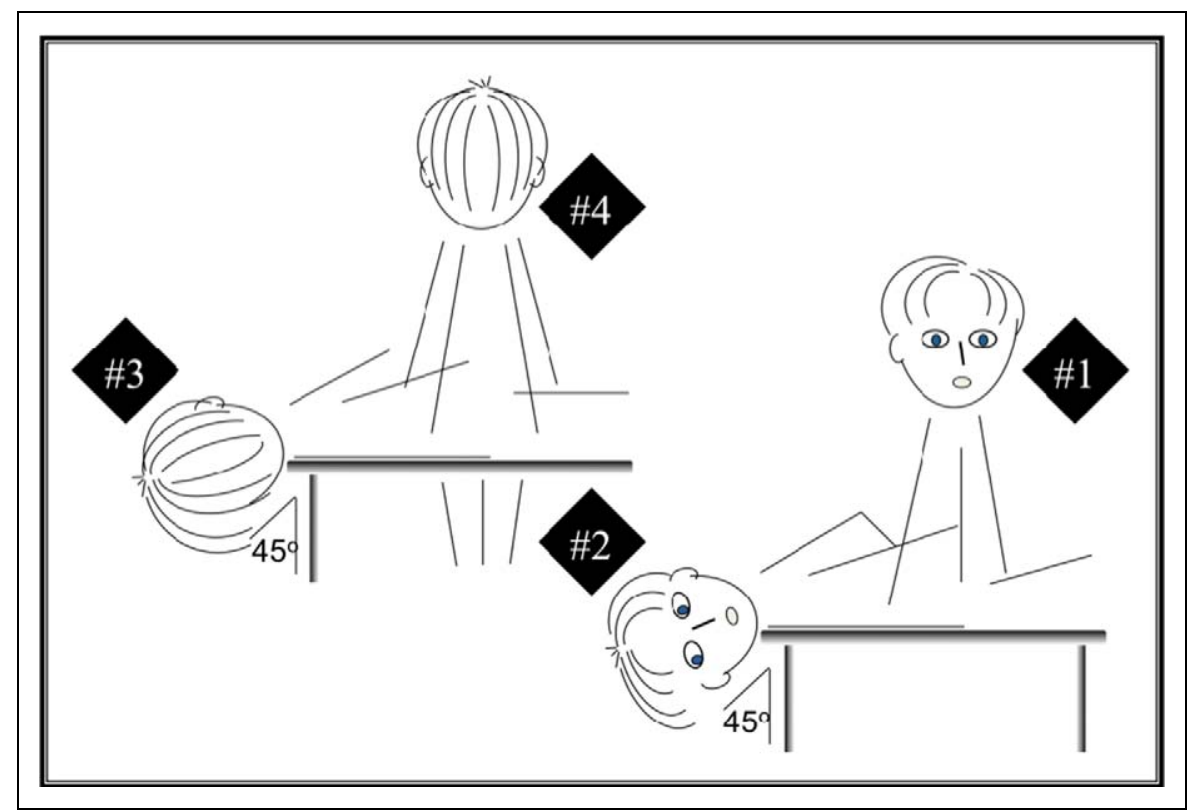

Figure I. Steps I and 2 represent the Dix-Hallpike maneuver which is used to diagnose BPPV of the posterior canal. Steps 3 and 4 are the canalith repositioning maneuver used to treat BPPV. BPPV, benign paroxysmal positional vertigo.

toward the affected ear. This is less effective than the modified Epley maneuver but can be repeated serially until the vertigo spells resolve. Brandt-Daroff exercises may be helpful in individual patients but there are insufficient studies to support their general use. A thorough description and videos of these maneuvers was presented by Fife et al in $2008 .^{26}$

\section{Meniere Disease}

Meniere disease, which is the eponymous designation for endolymphatic hydrops is due to abnormal fluid dynamics in the inner ear that cause the endolymphatic space to become congested. The association is far from perfect, however, as a large temporal bone bank series showed that patients can have pathological evidence of hydrops without a clinical history typical of Meniere disease. ${ }^{27}$ The hydrops is probably an end result of a variety of insults to the inner ear, including metabolic, vascular, inflammatory, and ischemic, though most cases appear to be idiopathic. Guidelines for the diagnosis of endolymphatic hydrops were developed by the American Academy of Otolaryngology, Head and Neck Surgery in 1995. A certain diagnosis requires histology and is therefore rarely obtained. A definite diagnosis requires 2 episodes of vertigo lasting at least 20 minutes with audiologically confirmed hearing loss as well as aural symptoms of tinnitus and/or ear fullness. ${ }^{28}$ The true prevalence and incidence of the disorder is difficult to ascertain since most epidemiological studies were performed before the current criteria. However, a telephone interview-based study in Germany found a population prevalence of $0.12 \%$ just based on symptoms and not on examinations or testing. ${ }^{29}$
Hearing loss in Meniere disease is gradual, with an initial low-frequency loss being classic. However, it can present with all patterns of hearing loss. Any patient who presents with sudden deafness and vertigo should not be diagnosed with Meniere disease. Such a patient should be considered to have had a vascular event until proven otherwise. Labyrinthitis can also present with rather sudden onset of vertigo and hearing loss, but it is generally over several minutes or even hours, and not hyperacute like a vascular event.

The tinnitus of Meniere is often described as a low-pitched, roaring tinnitus, which distinguishes it from the more common high-pitched tinnitus associated with age-related highfrequency sensorineural hearing loss. Most spells of Meniere disease last on the order of hours. The vertigo is usually severe and patients can develop prodromal symptoms of increasing ear fullness or tinnitus before the vertigo starts. There has also been a long recognized association between Meniere disease and migraine. Migrainous features such as headache, photophobia, and even aura have been reported to be quite prevalent during Meniere attacks. ${ }^{30}$ Meniere attacks can occur spontaneously or be triggered by high-salt-containing foods or stress. During the acute phase, the patient will experience decreased hearing in only 1 ear; concurrent bilateral hearing loss is uncommon. However, after 10 years, about $35 \%$ of patients can have the second ear affected. ${ }^{31}$ In the attack, the patient exhibits nystagmus that is either mixed horizontal torsional or vertical torsional, indicating the general involvement of all the semicircular canals. Depending on the phase of the vertigo in which the patient is seen, the nystagmus may beat toward the affected ear or beat away from the affected ear. As the nystagmus is peripheral in origin, it is easily suppressed by visual fixation. The nystagmus can be unmasked using either Frenzel 


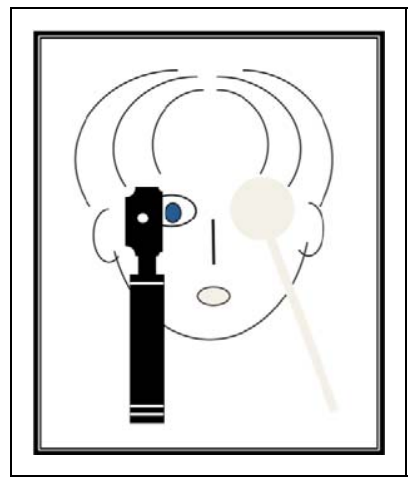

Figure 2. Occlusive ophthalmoscopy: Visualization of one fundus with an ophthalmoscope while occluding the other eye will effectively remove the ability of the patient to fixate and will uncover nystagmus caused by a peripheral vestibular lesion that is otherwise suppressed by fixation.

lenses (20 diopter lenses that prevent fixation) or a technique called occlusive ophthalmoscopy. In this technique, the examiner observes the optic disc of 1 eye with the ophthalmoscope while covering the other eye (Figure 2). Fixation is removed since both eyes are essentially occluded. It should be noted that since the back of the eye is being observed, the direction of the nystagmus observed will be in the opposite direction of the front of the eye.

In the acute setting, the goal is to treat the vertigo with vestibular suppressants such as meclizine, promethazine, and benzodiazepines and to maintain hydration. Vestibular suppressants should not be continued once the acute vertigo episode has stopped and should only be used on an "as needed" basis. Appropriate first-line preventive treatment for Meniere disease are the initiation of a diuretic such as hydrochlorothiazide ( $25 \mathrm{mg}$ daily) or acetazolamide (titrated to $250 \mathrm{mg}$ twice daily) and starting a low-salt diet $(<2 \mathrm{~g} / \mathrm{d})$. However, there are no good randomized placebo-controlled trials to support their use in Meniere disease. ${ }^{32}$

Baseline hearing decreases with time in the majority of patients but total deafness is unusual. ${ }^{33}$ If vertigo spells are frequent despite medical management, then surgical options should be explored with an otolaryngologist.

\section{Vestibular Neuritis}

This disorder is presumed to be due to reactivation of latent herpes simplex I in the vestibular ganglion, akin to Bell Palsy. ${ }^{34}$ The annual incidence is estimated at 3.5 per $100000 .{ }^{35}$ Patients with vestibular neuritis experience severe continuous vertigo, are extremely nauseated, and often vomit. In the acute phase, they will exhibit a spontaneous nystagmus with the fast phase beating toward the opposite ear. However, higher order ocular control centers will quickly suppress the nystagmus, and beyond the second day, nystagmus may only be seen by removing fixation such as by occlusive ophthalmoscopy (Figure 2) or with Frenzel lenses. Hearing and all neurological function should otherwise be normal. Vestibular function testing will show either a caloric paresis or paralysis in the affected ear with a gradual return of function as the patient recovers. An important bedside test is the head impulse test, which can help distinguish a vestibular neuritis from a cerebellar infarct, the other main cause of isolated acute vertigo. ${ }^{36}$

The head impulse test is a test of the vestibular-ocular reflex (VOR). In an awake patient, the test has to be performed with high acceleration movements because the VOR is maximally efficient at high accelerations, and the patient will be able to anticipate the examiner's motions to voluntarily move their eyes if the movements are too slow. To perform the head impulse test, the examiner faces the patient, holds the patient's head with both hands, and performs rapid small amplitude, high-acceleration movements while the patient is instructed to fixate on the examiner's nose (Figure 3). When the VOR is intact, thrusting the head to the side will cause an immediate compensatory conjugate eye movement in the opposite direction such that the patient maintains fixation on the examiner at all times. However, if the VOR is slowed by an inflamed demyelinated nerve, the eyes will transiently move with the head and the patient will have to make a saccade back to midline to maintain fixation on the examiner. ${ }^{37}$ Therefore, the examiner is looking for corrective saccades; the side to which the head is thrusted is the side being stimulated. Patients with acute cerebellar infarcts will have a normal head impulse test, as this is a test of a local brain stem arc. ${ }^{36}$

The role of steroids in the treatment of vestibular neuritis is still controversial. Several studies have shown that steroids given within the first 3 days of symptoms can improve vestibular function testing but it is not clear whether they improve functional outcome. ${ }^{38,39}$ Unlike acute facial palsy, in which there is now good evidence that steroids can improve functional recovery, there is no clear evidence that patients with vestibular neuritis are clinically improved with steroids. The hallmark difference between recovery of facial nerve function and vestibular nerve function is that a 1-sided vestibular paresis is well compensated for by the opposite vestibular system. Therefore, other risk factors for high-dose steroids should be considered when weighing the potential benefits.

As with Meniere disease, patients with vestibular neuritis should be treated with vestibular suppressants only during the acute vertiginous phase. Continuous use of vestibular suppressants can hinder central compensation and prolong the recovery period. Vestibular rehabilitation may also improve the recovery process. Patients with vestibular neuritis generally make an excellent functional recovery and recurrences are extremely rare. Therefore, "recurrent vestibular neuritis" should only be diagnosed in rare circumstances and with laboratory evidence of a new onset of vestibular paresis. The reported recurrence rate with an average of 5 to 7 years of follow-up is about $2 \%$ with the recurrences happening in either ear. ${ }^{6,40}$ Nonspecific dizziness and chronic anxiety can follow an acute episode, however. ${ }^{41}$ 


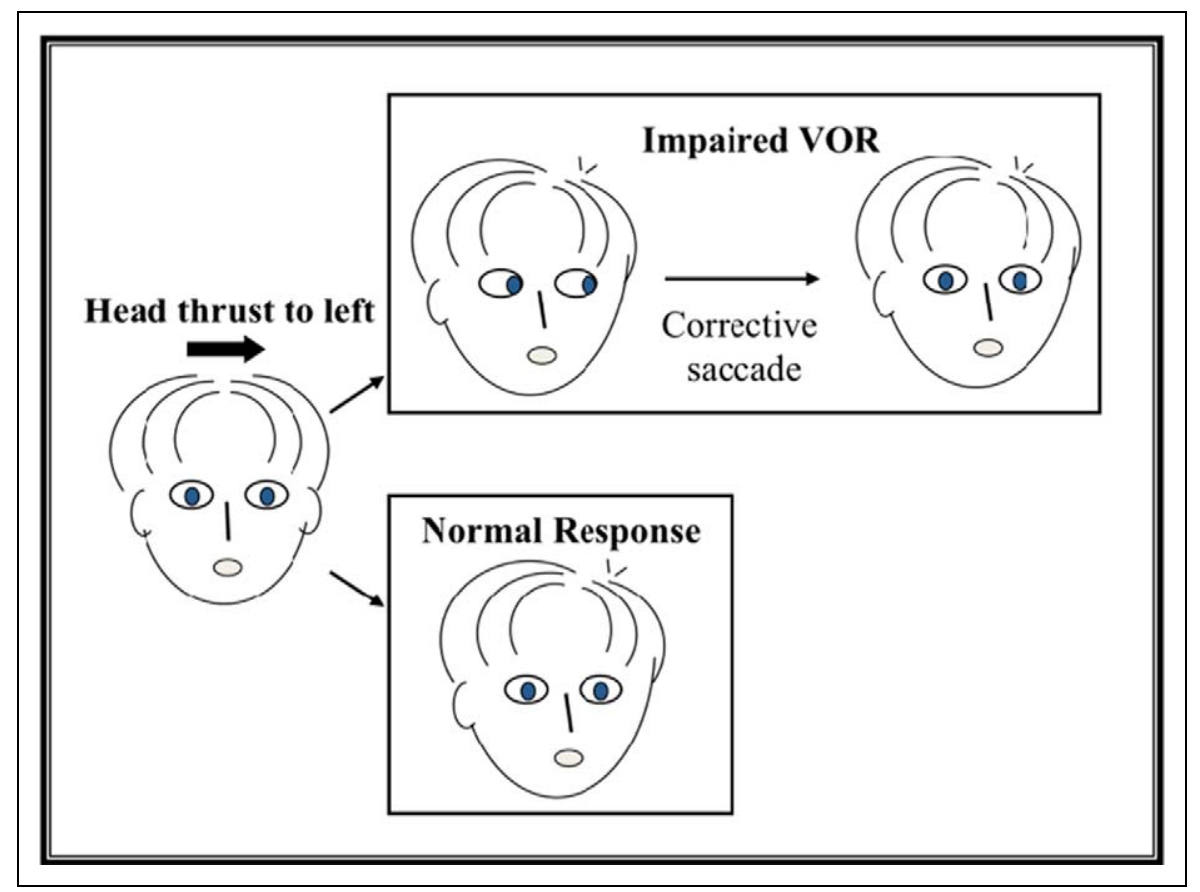

Figure 3. Head impulse test: Rapid high-acceleration head thrusts are performed (in this example, to the left) to elicit corrective saccades that reflect slowing of the vestibular-ocular reflex (VOR).

\section{Migrainous Vertigo}

Episodic vertigo spells occur in close to $30 \%$ of patients with migraine headache but usually follow the onset of the headaches by many years and sometimes start when the migraine headaches are improving. ${ }^{42,43}$ The association may thus not be initially apparent. Patients can experience a variety of combinations of headache and vertigo, with most patients experiencing some vertigo episodes with their typical headaches and others completely in isolation. ${ }^{44}$ The localization of vertigo in migraine is unknown as careful eye movement recordings during acute attacks can have both central and peripheral features. ${ }^{45}$ One suggestion that the origin may be peripheral is that migraineurs have a higher rate of baseline vestibular dysfunction than nonmigraineurs. ${ }^{46}$ Support for a central mechanism includes vertigo spells associated with typical aura and severe vertigo that is not associated with nystagmus. Some patients may report that their symptoms are worse with certain head positions, but this should not be confused with BPPV, since patients with migrainous vertigo will be nauseated, photophobic, or phonophobic during attacks. These patients respond well to regular migraine preventive medications and can be treated with vestibular suppressants in the acute setting.

\section{Ischemia}

Although ischemia to any portion of the vestibular pathway can cause vertigo, infarcts in the territory of the anterior inferior cerebellar artery (AICA) and the medial branch of the posterior inferior cerebellar artery (mPICA) are the most likely to be confused for inner ear disorders. Rules of thumb in lateralizing the side of infarction such as "falling to the side of the infarct," are not sensitive enough to localize the lesion. ${ }^{36}$ It is generally considered that patients with central disorders experience less prominent vertigo than those with peripheral disorders, but this is not specific enough to guide a diagnostic workup.

Anterior inferior cerebellar artery territory infarcts should be considered whenever a patient presents with the hyperacute onset of unilateral deafness and vertigo. Since the internal auditory artery branches from the AICA, patients with AICA infarcts can show a combination of peripheral and central abnormalities, including a vestibular paresis and a positive head impulse test. Central abnormalities can occur because the AICA serves the middle cerebellar peduncle, anterior cerebellar hemispheres, and lateral inferior pons. Stuttering onset of vertigo in a patient with known vascular risk factors should also prompt an immediate evaluation for posterior circulation insufficiency, particularly basilar artery stenosis in the vicinity of the take off of the AICA. ${ }^{47}$ In the largest series to date on the audiovestibular symptoms of AICA infarcts (82 patients), 98\% experienced vertigo, $96 \%$ showed abnormal central ocular features, $65 \%$ a vestibular paresis, and $63 \%$ hearing loss on presentation. Only 1 patient had auditory and vestibular loss without any central neurological signs. 48

Cerebellar infarction in the distribution of the MPICA can lead to isolated vertigo without auditory symptoms. The infarct is in the caudal cerebellum just off the midline. Central ocular features such as gaze-evoked nystagmus, poor smooth 
pursuit, and poor fixation-suppression of nystagmus should be carefully sought but some patients with mPICA infarcts can exhibit unidirectional beating nystagmus. A head impulse test should always be performed in such patients to confirm a normal VOR. In the acute setting, this is the only bedside test that can reliably distinguish an mPICA infarct from vestibular neuritis when the cerebellar examination is otherwise normal. $^{36}$

The decision to order neuroimaging in patients with vertigo rests mainly with the comfort of the practitioner in being able to rule-in one of the more common peripheral causes of vertigo. However, neuroimaging with magnetic resonance imaging (MRI) and either an MR or computed tomography (CT) angiogram should strongly be considered in the following circumstances: presentation of persistent vertigo with a hyperacute onset and a normal head impulse test; a first time presentation of vertigo with headache; vertigo with sudden deafness; vertigo with any central ocular motor signs or neurologic deficits, vertigo with cognitive changes; and the stuttering onset of vertigo spells (seconds to minutes) that does not fit the criteria for any peripheral vestibular disorder.

\section{Vestibular Paroxysmia}

Brief attacks of position-triggered vertigo can also be due to vestibular paroxysmia, which is the vestibular kin to trigeminal neuralgia. Patients experience seconds to minutes long episodes of vertigo that are often associated with tinnitus which can be triggered by very specific head or body positions. The Dix-Hallpike maneuver is negative in this disorder, but nystagmus can be uncovered by hyperventilation. It is treated with activity-dependent sodium channel blocking medications like carbamazepine and oxcarbazepine. ${ }^{49}$

\section{"Third-Window" Syndromes}

Less common conditions that may be encountered in the hospital setting are third-window syndromes like perilymphatic fistulas and superior canal dehiscence. ${ }^{50,51}$ The oval window and the round window are the only normal nonbony boundaries between the middle and inner ear. Third window refers to an abnormal third connection to the inner ear, such that now sound and pressure energy can be transmitted directly into the inner ear. The hallmark feature of these disorders are soundand pressure- (eg, coughing, Valsalva) induced attacks of vertigo. They are very brief but can occur multiple times a day. A history of vertigo attacks that are associated with middle ear surgery, recurrent middle ear infections, a cholesteatoma, or barotrauma such as blows to the ear or even forceful sneezing or straining should be sought. Perilymphatic fistulas may have symptoms similar to Meniere disease such as fluctuating hearing loss and are very difficult to diagnose even with exploratory middle ear surgery. Such patients should be evaluated with an audiogram and high-resolution CT of the temporal bones. A special test of saccule function called a vestibular- evoked myogenic potential (VEMP) can be helpful in the diagnosis of these dehiscence syndromes.

\section{Conclusion}

Acute vestibulopathies can be frightening for the patient, but practitioners who are at the frontlines in managing these patients can make a tremendous difference in relieving symptoms and starting the patient along the correct diagnostic pathway. A careful history that accurately details the important features of the vertigo-duration, chronicity, associated symptoms, and triggers should narrow down the differential quite effectively. A few simple bedside examinations can rule-in a benign diagnosis. If ambiguity or worrisome features exist, then further testing with neuroimaging, vestibular function, and auditory testing are required. However, an overreliance on these diagnostic tests can lead to confusion due to their nonspecificity and high rate of false positives and incidental findings. As in all good medicine, the practitioner needs to start with a good working differential diagnosis that is based on an excellent history and neurological examination.

\section{Declaration of Conflicting Interests}

The author declared no conflicts of interest with respect to the authorship and/or publication of this article.

\section{Funding}

Supported by grants R03DC010451 and U54RR019482.

\section{References}

1. von Brevern M, Radtke A, Lezius F, et al. Epidemiology of benign paroxysmal positional vertigo: a population based study. J Neurol Neurosurg Psychiatry. 2007;78(7):710-715.

2. Lopez-Escamez JA, Gamiz MJ, Finana MG, Perez AF, Canet IS. Position in bed is associated with left or right location in benign paroxysmal positional vertigo of the posterior semicircular canal. Am J Otolaryngol. 2002;23(5):263-266.

3. Tanimoto H, Doi K, Nishikawa T, Nibu K. Risk factors for recurrence of benign paroxysmal positional vertigo. J Otolaryngol Head Neck Surg. 2008;37(6):832-835.

4. Uneri A. Migraine and benign paroxysmal positional vertigo: an outcome study of 476 patients. Ear Nose Throat J. 2004; 83(12):814-815.

5. Ishiyama A, Jacobson KM, Baloh RW. Migraine and benign positional vertigo. Ann Otol Rhinol Laryngol. 2000;109(5): 377-380.

6. Mandala M, Santoro GP, Awrey J, Nuti D. Vestibular neuritis: recurrence and incidence of secondary benign paroxysmal positional vertigo. Acta Otolaryngol. 2010;130(5):565-567.

7. Welling DB, Parnes LS, O'Brien B, Bakaletz LO, Brackmann DE, Hinojosa R. Particulate matter in the posterior semicircular canal. Laryngoscope. 1997;107(1):90-94. 
8. Parnes LS, McClure JA. Free-floating endolymph particles: a new operative finding during posterior semicircular canal occlusion. Laryngoscope. 1992;102(9):988-992.

9. Cakir BO, Ercan I, Cakir ZA, Civelek S, Sayin I, Turgut S. What is the true incidence of horizontal semicircular canal benign paroxysmal positional vertigo? Otolaryngol Head Neck Surg. 2006; 134(3):451-454.

10. Prokopakis EP, Chimona T, Tsagournisakis M, et al. Benign paroxysmal positional vertigo: 10-year experience in treating 592 patients with canalith repositioning procedure. Laryngoscope. 2005;115(9):1667-1671.

11. Korres S, Balatsouras DG, Kaberos A, Economou C, Kandiloros D, Ferekidis E. Occurrence of semicircular canal involvement in benign paroxysmal positional vertigo. Otol Neurotol. 2002;23(6):926-932.

12. Zee DS, Leigh RJ. The Neurology of Eye Movements. 4th ed. New York, NY: Oxford University Press; 2006.

13. Cohen HS, Sangi-Haghpeykar H. Nystagmus parameters and subtypes of benign paroxysmal positional vertigo. Acta Otolaryngol. 2010;130(9):1019-1023.

14. Honrubia V, House M. Mechanism of posterior semicircular canal stimulation in patients with benign paroxysmal positional vertigo. Acta Otolaryngol. 2001;121(2):234-240.

15. Casqueiro JC, Ayala A, Monedero G. No more postural restrictions in posterior canal benign paroxysmal positional vertigo. Otol Neurotol. 2008;29(5):706-709.

16. Fyrmpas G, Rachovitsas D, Haidich AB, et al. Are postural restrictions after an Epley maneuver unnecessary? First results of a controlled study and review of the literature. Auris Nasus Larynx. 2009;36(6):637-643.

17. Brandt T, Huppert D, Hecht J, Karch C, Strupp M. Benign paroxysmal positioning vertigo: a long-term follow-up (6-17 years) of 125 patients. Acta Otolaryngol. 2006;126(2): 160-163.

18. Karlberg M, Hall K, Quickert N, Hinson J, Halmagyi GM. What inner ear diseases cause benign paroxysmal positional vertigo? Acta Otolaryngol. 2000;120(3):380-385.

19. Gordon CR, Levite R, Joffe V, Gadoth N. Is posttraumatic benign paroxysmal positional vertigo different from the idiopathic form? Arch Neurol. 2004;61(10):1590-1593.

20. Imai $T$, Ito $M$, Takeda $N$, et al. Natural course of the remission of vertigo in patients with benign paroxysmal positional vertigo. Neurology. 2005;64(5):920-921.

21. Herdman SJ, Tusa RJ. Complications of the canalith repositioning procedure. Arch Otolaryngol Head Neck Surg. 1996; 122(3):281-286.

22. De la Meilleure G, Dehaene I, Depondt M, Damman W, Crevits L, Vanhooren G. Benign paroxysmal positional vertigo of the horizontal canal. J Neurol Neurosurg Psychiatry. 1996;60(1):68-71.

23. Gufoni M, Mastrosimone L, Di Nasso F. Repositioning maneuver in benign paroxysmal vertigo of horizontal semicircular canal (in Italian). Acta Otorhinolaryngol Ital. 1998;18(6): 363-367.

24. Lempert T, Tiel-Wilck K. A positional maneuver for treatment of horizontal-canal benign positional vertigo. Laryngoscope. 1996;106(4):476-478.
25. Lempert T, Wolsley C, Davies R, Gresty MA, Bronstein AM. Three hundred sixty-degree rotation of the posterior semicircular canal for treatment of benign positional vertigo: a placebocontrolled trial. Neurology. 1997;49(3):729-733.

26. Fife TD, Iverson DJ, Lempert T, et al. Practice parameter: therapies for benign paroxysmal positional vertigo (an evidence-based review): report of the Quality Standards Subcommittee of the American Academy of Neurology. Neurology. 2008;70(22): 2067-2074.

27. Merchant SN, Adams JC, Nadol JB Jr. Pathophysiology of Meniere's syndrome: are symptoms caused by endolymphatic hydrops? Otol Neurotol. 2005;26(1):74-81.

28. Committee on Hearing and Equilibrium guidelines for the diagnosis and evaluation of therapy in Meniere's disease. American Academy of Otolaryngology-Head and Neck Foundation, Inc. Otolaryngol Head Neck Surg. 1995;113(3):181-185.

29. Radtke A, von Brevern M, Feldmann M, et al. Screening for Meniere's disease in the general population-the needle in the haystack. Acta Otolaryngol. 2008;128(3):272-276.

30. Radtke A, Lempert T, Gresty MA, Brookes GB, Bronstein AM, Neuhauser H. Migraine and Meniere's disease: is there a link? Neurology. 2002;59(11):1700-1704.

31. Huppert D, Strupp M, Brandt T. Long-term course of Meniere's disease revisited. Acta Otolaryngol. 2010;130(6):644-651.

32. Thirlwall AS, Kundu S. Diuretics for Meniere's disease or syndrome. Cochrane Database Syst Rev. 2006;3(1):CD003599.

33. Friberg U, Stahle J, Svedberg A. The natural course of Meniere's disease. Acta Otolaryngol Suppl. 1984;406(1):72-77.

34. Baloh RW, Ishyama A, Wackym PA, Honrubia V. Vestibular neuritis: clinical-pathologic correlation. Otolaryngol Head Neck Surg. 1996;114(4):586-592.

35. Sekitani T, Imate Y, Noguchi T, Inokuma T. Vestibular neuronitis: epidemiological survey by questionnaire in Japan. Acta Otolaryngol Suppl. 1993;503(1):9-12.

36. Lee H, Sohn SI, Cho YW, et al. Cerebellar infarction presenting isolated vertigo: frequency and vascular topographical patterns. Neurology. 2006;67(7):1178-1183.

37. Halmagyi GM, Curthoys IS, Cremer PD, Henderson CJ, Staples M. Head impulses after unilateral vestibular deafferentation validate Ewald's second law. J Vestib Res. 1990;1(2): 187-197.

38. Strupp M, Zingler VC, Arbusow V, et al. Methylprednisolone, valacyclovir, or the combination for vestibular neuritis. $N$ Engl J Med. 2004;351(4):354-361.

39. Shupak A, Issa A, Golz A, Margalit K, Braverman I. Prednisone treatment for vestibular neuritis. Otol Neurotol. 2008; 29(3):368-374.

40. Huppert D, Strupp M, Theil D, Glaser M, Brandt T. Low recurrence rate of vestibular neuritis: a long-term follow-up. Neurology. 2006;67(10):1870-1871.

41. Godemann F, Siefert K, Hantschke-Bruggemann M, Neu P, Seidl R, Strohle A. What accounts for vertigo one year after neuritis vestibularis - anxiety or a dysfunctional vestibular organ? J Psychiatr Res. 2005;39(5):529-534.

42. Brantberg K, Trees N, Baloh RW. Migraine-associated vertigo. Acta Otolaryngol. 2005;125(3):276-279. 
43. Kayan A, Hood JD. Neuro-otological manifestations of migraine. Brain. 1984;107(Pt 4):1123-1142.

44. Cha YH, Lee H, Santell LS, Baloh RW. Association of benign recurrent vertigo and migraine in 208 patients. Cephalalgia. 2009;29(5):550-555.

45. von Brevern M, Zeise D, Neuhauser H, Clarke AH, Lempert T. Acute migrainous vertigo: clinical and oculographic findings. Brain. 2005;128(2):365-374.

46. Bir LS, Ardic FN, Kara CO, Akalin O, Pinar HS, Celiker A. Migraine patients with or without vertigo: comparison of clinical and electronystagmographic findings. J Otolaryngol. 2003; 32(4):234-238.
47. Kim JS, Lee H. Inner ear dysfunction due to vertebrobasilar ischemic stroke. Semin Neurol. 2009;29(5):534-540.

48. Lee H, Kim JS, Chung EJ, et al. Infarction in the territory of anterior inferior cerebellar artery: spectrum of audiovestibular loss. Stroke. 2009;40(12):3745-3751.

49. Hufner K, Barresi D, Glaser M, et al. Vestibular paroxysmia: diagnostic features and medical treatment. Neurology. 2008; 71(13):1006-1014.

50. Minor LB. Clinical manifestations of superior semicircular canal dehiscence. Laryngoscope. 2005;115(10):1717-1727.

51. Minor LB. Labyrinthine fistulae: pathobiology and management. Curr Opin Otolaryngol Head Neck Surg. 2003;11(5): 340-346. 